\title{
Escalating threat from tuberculosis: the third epidemic
}

\author{
Adam S Malin, Keith P W J McAdam \\ Department of Clinical Sciences, London School of Hygiene and Tropical Medicine, \\ London, UK; Medical Research Council Laboratories at Fajara, The Gambia, Africa
}

Introductory article

\section{The third epidemic - multidrug-resistant tuberculosis}

\author{
K Neville, A Bromberg, R Bromberg, S Bonk, BA Hanna, WN Rom
}

We recently observed a striking increase in multidrug-resistant tuberculosis (MDR-TB) among patients admitted to the Chest Service at Bellevue Hospital Center in New York. We reviewed the laboratory susceptibility test results of 4681 tuberculosis (TB) cases over the past 20 years. Combined resistance to isoniazid and rifampin increased from 2.5\% in 1971 to $16 \%$ in 1991 with higher rates noted for individual drugs. We reviewed the medical records of 100 patients with drug-resistant TB, finding that these individuals were predominantly less than 40 years of age, minority, male, jobless, undomiciled, with a high percentage of drug abuse and human immunodeficiency virus infection. We conclude that the epidemics of AIDS and TB are complicated by a third epidemic of MDR-TB. This third epidemic requires urgent attention to achieve more rapid diagnosis, to develop new therapeutic regimens, and to address the social and hospital environment to care for these individuals. (Chest 1994;105:45-8)

\section{One disease - three epidemics}

THE FIRST EPIDEMIC: A GLOBAL RESURGENCE

The world faces three epidemics from tuberculosis. The first of these is the re-emergence of tuberculosis itself. Before 1985 the industrialised world had experienced a steady decline in tuberculosis. ${ }^{12}$ There had also been some success of tuberculosis control in the developing world with a decline in incidence in some countries and a stabilisation in others, ${ }^{3}$ albeit often at an unsatisfactory level. Overall, the pattern of events looked promising. Funding for control programmes in affluent countries was reduced, research interest waned, and it was believed that, like smallpox, this disease could be eventually eradicated. ${ }^{2}$ However, the disease has returned with a vengeance. In 1992 the World Health Organisation (WHO) declared tuberculosis a "global emergency". It estimated that 1.7 billion people were latently infected with $M y c o-$ bacterium tuberculosis (approximately one third of the world's population), with an annual toll of eight million new cases of active disease and 2.9 million deaths. Thus, tuberculosis represents the single biggest killer of all the infectious diseases and accounts for $26 \%$ of avoidable adult deaths in the developing world. ${ }^{1}$

THE SECOND EPIDEMIC: HIV AND TUBERCULOSIS Co-infection with HIV and tuberculosis is a "cruel duet", each exacerbating the other and resulting in a rapid progression of early HIV infection and spread of tuberculosis into the non-HIV infected population. ${ }^{4}$ This second epidemic of HIV-related tuberculosis has been termed the "new tuberculosis" and bears only a resemblance to its older sibling. The major differences are shown in the box. For example, pulmonary disease may present with non-specific features including radiographic bilateral lower zone shadowing, a loss of predilection for the upper lobes, and absence of cavities. Extrapulmonary tuberculosis, particularly disseminated disease, is more frequent. The age of presentation has shifted away from those previously most at risk - the very young and elderly - to young adults who account for those most economically productive. Of all the risk factors for the development of tuberculosis, HIV is by far the most potent with a relative risk of $6-100$ when

\section{Impact of HIV on tuberculosis}

Increase in reactivation of old latent infection

Increase in reinfection and recurrent disease following treatment

Loss of disease control in poor communities

Change in the clinical picture (increase in

extrapulmonary and disseminated disease)

Change in age of presentation (increase in young adults) Marked increase in mortality 
compared with HIV negative individuals. ${ }^{6}$ Not surprisingly, Africa is experiencing a major resurgence in tuberculosis, mainly as a consequence of the overlap between high levels of latent tuberculosis infection and a very high level of HIV infection, $20-67 \%$ of tuberculosis patients being co-infected with $\mathrm{HIV}^{7}$ In one large necroscopic study in Abidjan, Côte d'Ivoire, tuberculosis was found in over $50 \%$ of cadavers with AIDS-defining pathology. ${ }^{8}$ Particularly worrying is the fact that only one tenth of the world's tuberculosis infected population reside in Africa; a further billion live in Asia and the western Pacific. As HIV spreads into these countries, the potential outcome is devastating.

THE THIRD EPIDEMIC: MULTIDRUG RESISTANCE

The third epidemic is in our midst - namely, multidrugresistant tuberculosis. To date, the brunt of the epidemic has been confined to the USA, ${ }^{9-13}$ but the conditions that gave rise to multidrug-resistant tuberculosis exist throughout the world and we would be wise to learn from their experience. Of the US cities affected, New York has been particularly badly hit. This picture is well exemplified in the introductory article - a hospital-based study by Neville and colleagues in which cases of drugresistant tuberculosis seen at the Chest Service at Bellevue Hospital, New York are described. ${ }^{14}$ They reviewed 20 years of tuberculosis laboratory susceptibility tests in 4681 cases and observed a striking increase in multidrug-resistant tuberculosis. Combined resistance to isoniazid and rifampicin rose from $2.5 \%$ in 1971 to $16 \%$ in 1991 . Of those $16 \%$, over three quarters were resistant to four standard agents - rifampicin, isoniazid, streptomycin, and ethambutol. The clinical notes of 100 patients with drug-resistant tuberculosis were reviewed and compared with 54 randomly selected drug-sensitive tuberculosis patients. Demographic characteristics of both the drug-sensitive and drug-resistant groups were similar. Most of the patients belonged to one or more of the following groups: young (under 40 years), Black or Hispanic, unemployed, homeless, male, HIV-infected, or drug misuser. Clinical characteristics were also compared between drug-sensitive and drug-resistant groups. These features included: anergy, fever, cough, night sweats, weight loss, radiograph bilateral infiltrates, adenopathy, cavities, miliary shadowing, and normal chest radiograph. Only anergy appeared to be significantly more common in the drug-resistant group than the drug-sensitive group ( $33 \%$ versus $8 \%$; $<<0.05)$.

Recent experience and predictions for the future The pattern of drug resistance seen at Bellevue Hospital is reflected both in New York and the rest of the country. ${ }^{15}$ Overall, in the USA $3 \%$ of all new cases and $6.9 \%$ of recurrent cases were resistant to both rifampicin and isoniazid. ${ }^{15}$ Institutional outbreaks have been a particular problem, and the Centers for Disease Control (CDC) in Atlanta, Georgia has reported 22 such outbreaks. ${ }^{9}$ These have occurred in hospitals, ${ }^{121316}$ clinics, ${ }^{16}$ drug treatment centres, ${ }^{17}$ hospices and, prisons. ${ }^{1819}$ Those with HIV infection are especially vulnerable and transmission rates are high. ${ }^{20}$ Moreover, rapid progression and death within 1-4 months is the norm when there is coinfection with HIV. ${ }^{21}$ Those with- out HIV have a $50 \%$ treatment failure rate within $18-24$ months. ${ }^{922}$

Whilst most cases of multidrug-resistant tuberculosis have occurred throughout North America, evidence exists to suggest that rifampicin resistance may be increasing in Thailand and Tanzania. ${ }^{23}$ Moreover, significant drug resistance has been reported in West and Central Africa, with more than $30 \%$ primary resistance to isoniazid. ${ }^{23}$ Drug resistance to isoniazid in East Africa before HIV was stable at $7 \%$ for over 30 years. Data from a cohort in Kenya show no evidence of a subsequent increase. $^{6}$

\section{Why a resurgence now?}

SOCIOECONOMIC FACTORS - POVERTY AND A FAILING INFRASTRUCTURE

Earlier this century, before antituberculous chemotherapy, tuberculosis was decreasing in the industrialised world as a result of social and economic changes - for example, data from army recruits in the Netherlands suggested an annual rate of decline of $5-6 \%$. This improved to $10 \%$ with the introduction of effective drugs. Similar trends have been noted in US naval recruits up to the mid 1980s. The resurgence in tuberculosis in industrialised countries has been ascribed to: (1) immigration of a foreign population who are at high risk of developing disease, (2) coinfection with HIV, (3) an increase in specific high risk groups such as intravenous drug users, and (4) "other factors" not attributable to the above. Bloom and Murray analysed the relative contribution of such factors in the USA and showed that the increase cannot be entirely explained by the first three. ${ }^{1}$ They concluded that the resurgence is also due in part, to increasing poverty and, in particular, a failing tuberculosis programme infrastructure. All of these factors are present in a handful of US cities. New York exemplifies the plight of the urban poor leaving most of the country left relatively unscathed. This problem is depicted in the figure, which shows a doubling in tuberculosis case rates in New York but stability throughout the USA.

Techniques in molecular epidemiology (described on the next page) support this view. DNA fingerprinting has been used to distinguish new infections from reactivation (see box). Cluster cases, implicating recent trans-

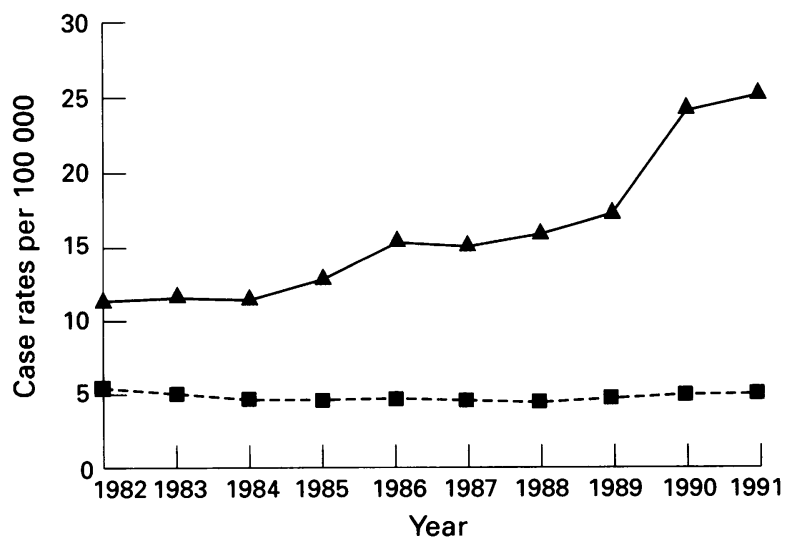

Tuberculosis case rates per 100000 in New York City $(\boldsymbol{\Delta})$ and the USA (D). Source: TB Control Program, BCDC NYS Department of Health. 


\section{Data ascertained by the use of molecular epidemiology (RFLP analysis)}

High rate of community transmission to $\mathrm{HIV}$-infected individuals ${ }^{243536}$

Risk factors for community transmission independent of HIV include minority groups, the young, and the poor $^{2436}$

A clustered isolate is more likely to be drug resistant ${ }^{24}$

Rapid progression to active disease in HIV-infected individuals ${ }^{20}$

High rates of institutional transmission to HIV-infected individuals ${ }^{20}$

Drug-resistant tuberculosis is readily transmitted among hospitalised patients with AIDS $^{1213}$

AIDS patients co-infected with tuberculosis can be superinfected with multidrug-resistant strains ${ }^{28}$

Non-HIV associated factors for new case clustering - for example, young, minority group, low income, drugresistant strain ${ }^{24}$

Relapse/reactivation or reinfection after treatment ${ }^{37}$

Identification of infection source ${ }^{38}$

Infection or reactivation (several studies in progress, e.g. migrant mine workers in South Africa originating from northern countries)

mission, were independently associated with a low household income and non-HIV infected Hispanics. ${ }^{24}$ Furthermore, using a multivariate regression model Brudney and Dobkin identified that non-compliance was associated with homelessness $(p<0.005)$ and alcoholism $(p<0.05)$, but not AIDS or AIDS-related complex $(p<0 \cdot 001) .{ }^{25}$ As well as the influence of poverty, a decaying tuberculosis programme infrastructure has also contributed to the development of multidrug-resistant tuberculosis. ${ }^{1}$ With the added burden of HIV, tuberculosis control programmes in the developing world are already overstretched. It is of vital importance that these are maintained.

\section{HOW SHOULD MUTIDRUG-RESISTANT TUBERCULOSIS BE SURVEYED?}

The Neville study raises wider issues associated with the surveillance of multidrug-resistant tuberculosis: (1) to what extent are these data skewed in favour of drug resistant cases (those with multidrug-resistant disease may circulate through several centres); (2) the data do not distinguish primary from acquired resistance; (3) no information is given describing the method of susceptibility testing; and (4) DNA fingerprinting was not assessed. The first three of these have been clearly reviewed in two recent articles from the WHO..$^{2326}$ From a public health perspective, WHO stresses the importance of identifying meaningful numerators and denominators when discussing both primary and acquired drug resistance rates. Of importance, when the acquired or secondary resistance rate is high, it indicates that the tuberculosis programme is functioning poorly in the current time. When primary or initial resistance rate is high, this indicates that the tuberculosis programme functioned poorly in the past. ${ }^{23}$. Thus, if surveys of multidrug-resistant tuberculosis are to be relevant, one must know the proportion of patients presenting for treatment who have previously received treatment.
Moreover, it is also useful to know the HIV serostatus. The WHO has put forward the following definitions in order to permit meaningful comparisons to be made.

Primary resistance means resistance to one or more drugs in a patient who has never received prior chemotherapy for tuberculosis. This group cannot be distinguished from those with undisclosed acquired resistance - that is, those patients who do not remember previous treatment, deny treatment, or where a treatment history is not asked. The initial resistance rate includes both of these groups in the numerator and can be defined simply as "the number of people never having received tuberculosis treatment with resistant bacilli divided by the number of new patients presenting for treatment".

The acquired resistance rate can be operationally defined using the numerator "all patients who begin treatment with susceptible bacilli and who become resistant six months later" and the denominator "the number of patients who started treatment with drug-sensitive bacilli". Such a definition permits evaluation of the programme and asks the important questions: "Of those who start treatment how many will develop drug-resistant disease"?

Multidrug resistance means resistant to at least rifampicin and isoniazid. These two drugs constitute the pivot of antituberculous chemotherapy, isoniazid being the main contributor to early antibactericidal activity and rifampicin offering excellent sterilising properties.

\section{MOLECULAR EPIDEMIOLOGY}

Restriction fragment length polymorphism (RFLP) is a powerful molecular technique which differentiates individual strains of $M$ tuberculosis. ${ }^{27}$ The technique takes advantage of naturally occurring insertion sequences of DNA (transposons) which randomly integrate into the genome of $M$ tuberculosis. The best delineated of these is IS6110 where 1-20 copies are arbitrarily dispersed throughout the mycobacterial chromosome. A restriction enzyme such as Pvu II will cleave this sequence wherever it occurs, and thus dissect the chromosome to produce a specific number of fragments each of a given size. Gel electrophoresis separates these fragments according to molecular weight and thus a specific banding pattern is produced. This pattern constitutes a unique DNA fingerprint. New cases caused by one strain that spread within a community will have the same fingerprint, whilst a "cluster" of cases suspected of being a consequence of reactivation will have different fingerprints thus precluding recent transmission. RFLP therefore represents a powerful epidemiological tool able to monitor outbreaks and identify risk factors associated with recent transmission (box).

Three recent studies from New York used RFLP to demonstrate clustering of multidrug-resistant tuberculosis ${ }^{121324}$ In one study 18 hospitalised AIDS patients with tuberculosis resistant to isoniazid and streptomycin were compared with AIDS patients with fully sensitive or single drug-resistant tuberculosis. The former group were considerably more likely to have been in hospital at the same time as a known drugresistant case (odds ratio $7 ; \mathrm{p}<0.01$ ) and to have shared the same ward (odds ratio 52; $p<0 \cdot 001$ ). Furthermore, DNA fingerprints were identical in 15 of 16 cases tested. 
Another study used RFLP analysis on isolates from 130 unselected tuberculosis patients over a three year period from a large hospital in the Bronx. Over a third of these cases showed clustering indicative of recent transmission. Independent risk factor analysis demonstrated that drug resistance was 4.5 times more likely in clustered groups $(\mathrm{p}<0.005) .{ }^{24}$ These studies and others ${ }^{1928}$ have clearly shown clustering of newly transmitted cases with primary drug-resistant tuberculosis. Moreover, it is likely that many patients and health care workers were infected (as defined by skin test conversion) with multidrug-resistant tuberculosis. ${ }^{1319}$ In most cases, guidelines for the management of cases of smear positive tuberculosis were not followed. ${ }^{121319}$

\section{Solutions}

What lessons can be learnt from the USA? HIV infection, drug misuse, poverty, and homelessness constitute key risk factors in the transmission of multidrugresistant tuberculosis. However, it is unlikely that we will be able to impinge upon these, at least in the near future. Intervention with alternative drugs that are presently available is unlikely to yield better results. In centres of excellence the cure rate for multidrug-resistant tuberculosis is hardly better than if the patient had received no treatment. ${ }^{19}$ As emphasised by Kochi and colleagues, multidrug-resistant tuberculosis is not a new problem. ${ }^{23}$ It first occurred in many industrialised countries in the 1950 s as a consequence of poor tuberculosis treatment programmes. At the time, $97 \%$ cure rates were possible with isoniazid, PAS, and streptomycin. However, the regimen was inadequately established. This resulted in acquired drug resistance, chronic cases, and transmission of drug-resistant tuberculosis into the community. The problem was successfully overcome, not by a change in drugs, but by an improvement in the application of the existing regimen.

\section{ADEQUATE INFRASTRUCTURE}

Poverty alone cannot account for failures in completion of treatment. Several cities in the USA have produced data indicating very poor treatment completion rates, ${ }^{1}$ whereas resource-poor countries such as Mozambique, Tanzania and Malawi have achieved high cure rates associated with a high percentage of treatment completion. $^{3}$ A well designed national tuberculosis programme receiving adequate funding is a prerequisite to effective control. ${ }^{29}$

\section{PRESCRIPTION OF COMBINED PREPARATIONS}

It has long been known that drug resistance rapidly develops in patients taking any single antituberculous drug. The spontaneous mutation rate for isoniazid is $10^{-6}$ per division per organism and, for rifampicin, it is $10^{-8}$. Development of combined resistance is multiplicative; in the case of isoniazid and rifampicin, $10^{-14}$. This number is far in excess of the bacillary load in most cases of tuberculosis (a tuberculous cavity will normally harbour $10^{7}-10^{9}$ organisms).$^{26}$ This is a strong argument in favour of using combined preparations. Cheaper versions of rifampicin and isoniazid are required for the developing world - preferably combined formulations. Although several combined formulations have been manufactured at low cost, there have been problems with bioavailability. This underlies the importance of checking bioavailability data whenever new combination regimens are contemplated.

\section{COMPLIANCE - INDUCEMENT OR ENFORCEMENT}

When an appropriate regimen is taken, more than $95 \%$ of organisms are killed within the first few weeks of therapy. However, a further prolonged period of treatment (at least a total of six months) is required to eradicate metabolically inactive persister bacilli. The patient usually feels well within a few weeks of treatment, thus making further adherence to therapy particularly difficult. Compliance and cure rates have been highest with short-course chemotherapy (six months) ${ }^{323}$ and when patients have been in hospital or have received directly observed therapy. ${ }^{112230-32}$ Hospitalisation may be reasonable for the period when the patient is infectious; beyond this, it is both expensive and enforced incarceration is ethically dubious. For most cases good compliance can be achieved using directly observed therapy.

\section{DIRECTLY OBSERVED THERAPY AND INTERMITTENT TREATMENT}

Drug compliance can be vastly improved if the patient is directly observed to take the medication. Excellent drug completion rates and high cure rates have been reported using directly observed therapy both in industrialised $^{2232}$ and developing ${ }^{3031}$ countries. A protocol for directly observed therapy employed in Denver, Colorado, used two weeks of daily therapy followed by 24 weeks of twice weekly intermittent therapy. ${ }^{22}$ Less than $10 \%$ of participants were lost to follow up. The added expense of the time of health care workers is compensated by savings from reduction in drug use and investigations (monitoring can be less rigorous with directly observed therapy). Moreover, the potential savings from eliminating new drug resistance is huge. Although many studies have used health care workers to perform this role, there is no reason why other responsible members of the community cannot do this. Wilkinson reports an innovative community-based study undertaken in KwaZulu, South Africa, where half the patients received directly observed therapy supervised by non-health care workers such as local shop keepers $^{31} ; 89 \%$ of surviving patients completed treatment, thus demonstrating the success of a cheap and innovative approach to tuberculosis control. Other countries have employed alternative strategies to improve compliance. These have included blister pack supplies of drugs and a deposit system with return on completion of treatment.

\section{PREVENTION OF NOSOCOMIAL SPREAD}

Most outbreaks result from poor infection control. ${ }^{1213}$ Patients and health care workers are exposed to the index case usually because of (1) failure to diagnose disease, (2) failure to confine untreated active tuberculosis, or (3) failure to diagnose/treat adequately multidrug-resistant tuberculosis. ${ }^{9}$ US guidelines issued by 
CDC in 1990 indicated a need to limit exposure from infectious droplet nuclei with the use of negative pressure isolation, six air changes per hour, and air disinfection using ultraviolet light. Some authorities have also advocated the use of respirators. Revised CDC guidelines from 1994 include the addition of high efficiency particulate air (HEPA) filters. However, there is little evidence to support the benefits of these expensive devices and such control measures require field evaluation. Adequate control measures should include isolation of a smear-positive case during the first two weeks of treatment - ideally with a commode, an anteroom, and negative pressure ventilation to the outside. There is probably little benefit in health care workers or visitors wearing masks unless they are filtered and tightly fitting. However, smear-positive cases should wear a simple surgical mask when leaving their rooms as a barrier to reduce droplet nuclei.

RAPID DIAGNOSIS OF DISEASE AND DRUG SUSCEPTIBILITY A diagnosis of tuberculosis can be suspected when a clinical sample stains positive for acid fast bacilli. However, whilst this is a useful indicator for initial management, a number of problems remain outstanding - for example, (1) increasing immune depression is associated with a higher incidence of smear-negative disease; (2) the presence of acid fast bacilli does not exclude atypical mycobacteria such as Mycobacterium avium-intracellulare (MAI) complex (although this latter diagnosis is unlikely in Africa); and (3) drug susceptibility cannot be deduced. Until recently, confirmation of the species and drug susceptibility has been very slow, usually $6-10$ weeks. However, in sophisticated laboratories rapid diagnosis and susceptibility testing can be achieved within 10-21 days (and perhaps even less) using radiometric culture (BACTEC), nucleic acid probes, and high performance liquid chromatography of mycolic acids. ${ }^{2}$ However, such facilities are not available in the developing world and, without dramatic modification, are not likely to be.

Even using a radiometric culture system, a patient with multidrug-resistant tuberculosis may remain effectively untreated for two weeks before a result is available. Jacobs and colleagues report a novel system for very rapid detection of the organism and drug susceptibility testing. Viable mycobacteria can be infected with reporter phages expressing the firefly luciferase gene resulting in photon release in only live organisms. The light goes out with the addition of an antituberculous drug to drug-sensitive but not drug-resistant strains. ${ }^{33}$ As yet the test is not sensitive enough to work with the small number of bacilli found in sputum samples. Moreover, reporter phages are not effective at colonising all mycobacterial strains.

TREATMENT FOR MULTIDRUG-RESISTANT TUBERCULOSIS In patients who are resistant to isoniazid and rifampicin the results of treatment are very poor. ${ }^{21}$ Second and third line drugs are considerably less effective and there is no immediate hope for the development of new drugs. Protocols for the treatment of these patients have been outlined by the $\mathrm{CDC}^{15}$ and elsewhere. ${ }^{1}$ It is a mistake just to add another drug when a patient is not responding clinically, as this will increase the catalogue of drugs to which resistance is acquired. Where multidrug-resistant tuberculosis is suspected, the patient should be started on five or six drugs. When results of susceptibility testing are available, at least three drugs to which the strain is susceptible should be continued for up to 24 months, depending on the severity of resistance. Iseman has reviewed the treatment of multidrug-resistant tuberculosis and suggests several regimens for patients with

\section{LEARNING POINTS}

* The incidence of multidrug-resistant tuberculosis is increasing rapidly in underprivileged, uncompliant, minority groups in developed countries in whom it is often fatal.

* Multidrug-resistant tuberculosis already poses an additional and serious threat to those in close contact through social and health care occupations.

* As worldwide trends continue towards increasing incidence of tuberculosis and HIV infection, the problem from multidrug-resistant disease is likely to increase and to pose a future threat to all communities.

* It is largely a consequence of recent failings in the infrastructure of tuberculosis control, and of inappropriate treatment in poorly compliant subjects.

* Its control will require rigorous control of tuberculosis itself, using novel strategies in the poorly compliant so that the continued emergence of multidrug-resistant organisms is minimised.

* The development of new drugs offers less hope of an immediate solution.

* The use of newly developed molecular techniques will improve rapid recognition of multidrug-resistant tuberculosis so that delay in appropriate treatment with multiple drugs may be minimised. 
various patterns of drug resistance. ${ }^{11}$ Chemoprophylaxis for those exposed to multidrug-resistant tuberculosis is difficult. Most data supporting the efficacy of chemoprophylaxis in drug-sensitive strains come from using isoniazid. ${ }^{4}$ However, data from animal studies and on theoretical grounds indicate that both rifampicin and pyrazinamide should be effective. Ongoing trials in Africa and the USA may confirm this. A chemoprophylaxis regimen should be determined according to the pattern of drug resistance to which the contact is exposed. ${ }^{34}$ Rifampicin alone or two-drug combinations selected from rifampicin, pyrazinamide, ethambutol, and a fluoroquinolone (ciprofloxacin or ofloxacin) seem reasonable given our current knowledge. ${ }^{1}$ However, there are concerns about the use of single agent prophylaxis in a setting where tuberculosis may be difficult to diagnose, particularly in those dually infected with HIV. In the presence of moderate to large bacterial loads, induction of drug resistance poses a realistic danger.

\section{Conclusions}

Treatment for multidrug-resistant tuberculosis is, at best, highly unsatisfactory. Developing countries have either no or limited access to testing for drug susceptibility. Even if such a facility were available, the cost of treatment for multidrug-resistant disease is prohibitive and the only hope of overcoming it is prevention. ${ }^{29}$ This article has discussed several ways of improving compliance. Directly observed therapy, intermittent and supervised regimens, combined drug preparations, and other novel means such as immunotherapy, all require operational evaluation. It is essential to know how these interventions would work in a national tuberculosis control programme. Adequate funding must be made available to support such programmes and operational research. Furthermore, a global drug resistance surveillance system is required to monitor the effectiveness of control interventions. If we do not act now but wait to see if this third epidemic will spread, by the time we see it it will be too late.

1 Bloom BR, Murray CJ. Tuberculosis: commentary on a reemergent killer (see comments). Science 1992;257:1055-64

2 Hopewell PC. Impact of human immunodeficiency virus infection on the epidemiology, clinical features, management, and control of tuberculosis. Clin Infect Dis 1992;15:540-7.

3 Murray CJ, DeJonghe E, Chum HJ, Nyangulu DS, Salomao A, Styblo $K$. Cost effectiveness of chemotherapy for pulmonary tuberculosis in K. Cost effectiveness of chemotherapy for pulmon 1931;338:1305-8.

4 Pape JW, Jean SS, Ho JL, Hafner A, Johnson Jr WD. Effect of isoniazid prophylaxis on incidence of active tuberculosis and progression of HIV infection. Lancet 1993;342:268-72.

5 Snider Jr DE, Roper WL. The new tuberculosis (editorial; comment). $N$ Engl f Med 1992;326:703-5.

6 Nunn PP, Elliott AM, McAdam KPWJ. Impact of human immunodeficiency virus on tuberculosis in developing countries. Thorax 1994;49:511-8.

7 De Cock KM, Soro B, Coulibaly IM, Lucas SB. Tuberculosis and HIV infection in sub-Saharan Africa. $\mathscr{Y} A M A$ 1992;268:1581-7.

8 Lucas SB, Hounnou A, Peacock C, Beaumel A, Djomand G, N'Gbichi $\mathrm{JM}$, et al. The mortality and pathology of HIV infection in a west JM, et al. The mortality and pathology of HIV infy (see comments). AIDS 1993;7:1569-79.

9 Bennett D, Watson J. Tuberculosis in the immunocompetent patient. Curr Opin Infect Dis 1994;7:184-91.

10 Valway SE, Richards SB, Kovacovich J, Greifinger RB, Crawford JT, Dooley SW. Outbreak of multi-drug-resistant tuberculosis in a New York State prison, 1991. Am $\mathcal{F}$ Epidemiol 1994;140:113-22.
11 Iseman MD. Treatment of multidrug-resistant tuberculosis. $N$ Engl $\mathcal{f}$ Med 1993;329:784-91.

12 Edlin BR, Tokars JI, Grieco MH, Crawford JT, Williams J, Sordillo $\mathrm{EM}$, et al. An outbreak of multidrug-resistant tuberculosis among hospitalized patients with the acquired immunodeficiency syndrome (see comments). N Engl f Med 1992;326:1514-21.

13 Pearson ML, Jereb JA, Frieden TR, Crawford JT, Davis BJ, Dooley SW, et al. Nosocomial transmission of multidrug-resistant Mycobacterium tuberculosis. A risk to patients and health care workers (see comments). Ann Intern Med 1992;117:191-6.

14 Neville K, Bromberg A, Bromberg R, Bonk S, Hanna BA, Rom WN. The third epidemic - multidrug-resistant tuberculosis. Chest 1994;105:45-8.

15 Centers for Disease Control. Initial therapy for tuberculosis in the era of multidrug resistance. Recommendations of the Advisory Council for the Elimination of Tuberculosis. MMWR 1993;42:1-8.

16 Centers for Disease Control. Nosocomial transmission of multidrugresistant tuberculosis to health-care workers and HIV-infected patients in an urban hospital - Florida. MMWR 1990;39:718-22.

17 Centers for Disease Control. Transmission of multidrug-resistant tuberculosis from an HIV-positive client in a residential substance-abuse treatment facility - Michigan. MMWR 1991;40:129-31.

18 Tartaglia J, Pincus S, Paoletti E. Poxvirus-based vectors as vaccine candidates. Crit Rev Immunol 1990;10:13-30.

19 Centers for Disease Control. Transmission of multidrug-resistant tuberculosis among immunocompromised persons in a correctional system - New York, 1991. MMWR 1992;41:507-9.

20 Daley CL, Small PM, Schecter GF, Schoolnik GK, McAdam RA, Jacobs Jr WR, et al. An outbreak of tuberculosis with accelerated progression among persons infected with the human immunodeficiency virus. An analysis using restriction-fragment-length polymorphisms. $N$ Engl f Med 1992;326:231-5.

21 Fischl MA, Daikos GL, Uttamchandani RB, Poblete RB, Moreno JN, Reyes RR, et al. Clinical presentation and outcome of patients with HIV infection and tuberculosis caused by multiple-drug-resistant bacilli. Ann Intern Med 1992;117:184-90.

22 Iseman MD, Cohn DL, Sbarbaro JA. Directly observed treatment of tuberculosis. We can't afford not to try it (see comments). N Engl $\mathcal{F}$ Med 1993;328:576-8.

23 Kochi A, Vareldzis B, Styblo K. Multidrug-resistant tuberculosis and its control. Res Microbiol 1993;144:104-10.

24 Alland D, Kalkut GE, Moss AR, McAdam RA, Hahn JA, Bosworth W, et al. Transmission of tuberculosis in New York City. An analysis by DNA fingerprinting and conventional epidemiological methods (see comments). N Engl f Med 1994;330:1710-6.

25 Brudney K, Dobkin J. Resurgent tuberculosis in New York City. Human immunodeficiency virus, homelessness, and the decline of tuberculosis control programs. Am Rev Respir Dis 1991;144:745-9.

26 Vareldzis BP, Grosset J, de Kantor I, Crofton J, Laszlo A, Felten M, et al. Drug-resistant tuberculosis: laboratory issues. Tuberc Lung Dis 1994;75:1-7.

27 Eisenach $\mathrm{KD}$. Use of an insertion sequence for laboratory diagnosis and epidemiologic studies of tuberculosis. Ann Emerg Med 1994;24: 450-3.

28 Horn DL, Hewlett Jr D, Haas WH, Butler WR, Alfalla C, Tan E, et al. Superinfection with rifampin-isoniazid-streptomycin-ethambutol (RISE)-resistant tuberculosis in three patients with AIDS: con(RISE)-resistant tuberculosis in three patients with AIDS: confirmation by polyr

29 McAdam KPWJ. Back to the future: 'the ten commitments'. In: Porter JDH, McAdam KPWJ, eds. Tuberculosis: back to the future. Chichester: John Wiley, 1994;267-76.

30 Kumaresan JA, Maganu ET. Case holding in patients with tuberculosis in Botswana. BMf 1992;305:340-1.

31 Wilkinson D. High-compliance tuberculosis treatment programme in a rural community. Lancet 1994;343:647-8.

32 Weis SE, Slocum PC, Blais FX, King B, Nunn M, Matney GB, et al. The effect of directly observed therapy on the rates of drug resistance The effect of directly observed therapy on the rates of drug resistance
and relapse in tuberculosis (see comments) $N$ Engl $\mathcal{F}$ Med 1994;330: and relapse

33 Jacobs Jr WR, Barletta RG, Udani R, Chan J, Kalkut G, Sosne G, et al. Rapid assessment of drug susceptibilities of Mycobacterium tuberculosis by means of luciferase reporter phages (see comments) Science 1993;260:819-22.

34 Centers for Disease Control. Management of persons exposed to multidrug-resistant tuberculosis. MMWR 1992;41:61-71.

35 Tabet SR, Goldbaum GM, Hooton TM, Eisenach KD, Cave MD, Nolan CM. Restriction fragment length polymorphism analysis detecting a community-based tuberculosis outbreak among persons tecting a community-based tuberculosis outbreak among persons infected

36 Small PM, Hopewell PC, Singh SP, Paz A, Parsonnet J, Ruston DC et al. The epidemiology of tuberculosis in San Francisco. A populationbased study using conventional. and molecular methods (see comments). N Engl f Med 1994;330:1703-9.

37 Hawken M, Nunn P, Gathua S, Brindle R, Godfrey Faussett P, Githui W, et al. Increased recurrence of tuberculosis in HIV-1-infected patients in Kenya. Lancet 1993;342:332-7.

38 Godfrey Faussett P, Mortimer PR, Jenkins PA, Stoker NG. Evidence of transmission of tuberculosis by DNA fingerprinting. BMf 1992 305:221-3. 